\title{
Identifying risk factors for cesarean scar pregnancy: a retrospective study of 79 cases
}

\author{
Min Shi ${ }^{1}$, Hui Zhang ${ }^{1}$, Shasha Qi ${ }^{1}$, Wenhui Liuㄹ, Ming Liu ${ }^{1}$, Xingbo Zhao ${ }^{1}$, Yulan Mu ${ }^{1}$ \\ ${ }^{1}$ Department of Obstetrics and Gynecology, Shandong Provincial Hospital Affiliated to Shandong University, Jinan, China \\ ${ }^{2}$ Information and Data Analysis Laboratory, School of Public Health, Shandong University, Jinan, China
}

\begin{abstract}
Objectives: To explore the possible risk factors for cesarean scar pregnancy (CSP), the incidence of which is increasing rapidly in China.

Material and methods: 79 patients with CSP and 69 non-CSP expectant mothers with at least 1 previous cesarean section were employed in the study. The obstetric histories of the participants were collected and analyzed using Chi square test. Results: We found that $77.2 \%$ CSP patients had $\geq 3$ pregnancies and only $36.2 \%$ women had $\geq 3$ pregnacies in non-CSP group. During the previous cesarean delivery, $21.5 \%$ of CSP patients had entered the first stage of labor, which was $43.5 \%$ in non-CSP group $(P<0.05)$. Cephalopelvic disproportion occurred in $51.9 \%$ of CSP patients, which was significantly higher than that $(23.2 \%)$ in non-CSP group $(P<0.01) .11 .4 \%$ of CSP patients had undergone cesarean section due to breech and shoulder presentation in the past, which was only $1.4 \%$ in non-CSP group. However, no significance was noted $(P>0.05)$. We did not find significant differences between the CSP and non-CSP patients in maternal age, multiple cesarean sections, gestational age, emergency or elective caesarean section.

Conclusions: Multiple pregnancies, absence of the first stage of labor, and cephalopelvic disproportion might be the risk factors for the occurrence of CSP.
\end{abstract}

Key words: cesarean scar pregnancy, cesarean section, lower uterine segment

Ginekologia Polska 2018; 89, 4: 196-200

\section{INTRODUCTION}

Ectopic pregnancy in caesarean section scars, which was first defined in 1978 [1], is a rare form of ectopic pregnancy $[2,3]$, located in the scar from a previous cesarean sectio [4], and it has become an important and serious problem over the last 10 years [5]. With the rise of the cesarean section rate [6] and the widespread use of transvaginal sonography, the incidence of cesarean scar pregnancy (CSP) is increasing rapidly $[7,8]$. As reported, the incidence of CSP was 1:1800-1:2226 [9], which was recurrent in some cases [10], representing $6.1 \%$ of all ectopic pregnancy [11]. The disease is usually life-threatening with a high risk for uterine rupture $[12]$ and fatal hemorrhage $[9,13]$. Since we have known that large uterine defects are known risk factors for scar dehiscence, the repair of the defect to reinforce the myometrial endurance seems to be an appropriate method of treatment [14], but not one repair surgery been suggest. It was badly needed to find the risk factors of CSP, since the earlier the recognition and diagnosis of this disease be aware of, the better the outcome would be [15].

Previous studies determined some risk factors for CSP Breech presentation caused non-developed lower uterine segment were involved in the occurrence of CSP [7, 8]; histories of endometrial or muscular injuries, including injuries caused by curettage, myomectomy, and hysteroscopic surgery, were correlated to a higher risk for CSP; adenomyosis, in vitro fertilization, and manual removal of placenta were also reported to be risk factors $[16,17]$. In addition, the surgical technique might also affect the occurrence of CSP [7].

The current study retrospectively analyzed the clinical information of the 79 patients with CSP in the Department of gynecology and obstetrics in Shandong Provincial Hospital affiliated to Shandong University and explored the possible risk factors for the occurrence of CSP. 


\section{MATERIAL AND METHODS \\ General information}

149 women who got repregnance after caesarean were included from 2011 to 2013 in Shandong Provincial Hospital, among whom 79 patients with CSP (CSP group) and 69 without CSP (non-CSP group). This study was conducted in accordance with the declaration of Helsinki and conducted with approval from the Ethics Committee of Shandong University with written informed consent was obtained from all participants.

\section{Inclusion criteria}

The inclusion criteria of CSP was according to the sonographic criteria proposed by Jurkovic et al and Vial et al. $[9,16]$ : (I) Doppler scans show functional trophoblastic circulation; (II) the trophoblast is mainly located between the bladder and anterior uterine wall; (III) the uterine cavity and cervical canal are empty; and (IV) thin or discontinuous myometrium between the gestational sac and bladder on a sagittal image of the uterus running through the amniotic sac. The exclusion was (I) any other uterine disease (like fibroid); (II) any congenital disease of the infant, (III) hematological disease, (IV) artificial insemination. All the clinical data of the medical history, including the general information, gathered on each participant included (Tab. 1): maternal age, chief complain, number of previous cesarean section, number of previous pregnancies, gestational age when the previous cesarean section was performed, emergency or elective surgery during the previous cesarean section,

Table 1. Information of the participants

\begin{tabular}{|c|c|c|c|c|c|c|c|c|}
\hline \multirow[b]{2}{*}{ Factors } & \multirow{2}{*}{\multicolumn{2}{|c|}{$\begin{array}{l}\text { Number of participants } \\
\text { (n) }\end{array}$}} & \multicolumn{2}{|c|}{ Percentage (\%) } & \multirow{2}{*}{\multicolumn{2}{|c|}{ Chi-square }} & \multirow{2}{*}{\multicolumn{2}{|c|}{$\mathbf{P}$}} \\
\hline & & & $\begin{array}{c}\text { CSP } \\
(n=79)\end{array}$ & $\begin{array}{c}\text { CTR } \\
(n=69)\end{array}$ & & & & \\
\hline \multicolumn{9}{|l|}{ Number of cesarean delivery } \\
\hline$\geq 2$ & 9 & 9 & 11.4 & 13.0 & \multirow{2}{*}{\multicolumn{2}{|c|}{0.094}} & \multirow{2}{*}{\multicolumn{2}{|c|}{0.759}} \\
\hline$<2$ & 70 & 60 & 88.6 & 87 & & & & \\
\hline \multicolumn{9}{|l|}{ Gestational age } \\
\hline$\geq 37$ weeks & 69 & 61 & 87.3 & 88.4 & \multirow{2}{*}{\multicolumn{2}{|c|}{0.039}} & \multirow{2}{*}{\multicolumn{2}{|c|}{0.843}} \\
\hline$<37$ weeks & 10 & 8 & 12.7 & 11.6 & & & & \\
\hline \multicolumn{9}{|l|}{ Number of pregnancy } \\
\hline$\geq 5$ & 18 & 3 & 22.8 & 4.3 & \multirow{4}{*}{\multicolumn{2}{|c|}{27.481}} & \multirow{4}{*}{\multicolumn{2}{|c|}{$<0.001 \mathrm{a}$}} \\
\hline 4 & 19 & 4 & 24.1 & 5.8 & & & & \\
\hline 3 & 24 & 18 & 30.4 & 26.1 & & & & \\
\hline 2 & 13 & 19 & 16.5 & 27.5 & & & & \\
\hline \multicolumn{9}{|l|}{ Previous cesarean delivery } \\
\hline Emergency surgery & 33 & 26 & 41.8 & 37.7 & \multirow{2}{*}{\multicolumn{2}{|c|}{0.257}} & \multirow{2}{*}{\multicolumn{2}{|c|}{0.612}} \\
\hline Elective surgery & 46 & 43 & 58.2 & 62.3 & & & & \\
\hline \multicolumn{9}{|l|}{ Entering the first stage of labor } \\
\hline Yes & 17 & 30 & 21.5 & 43.5 & \multirow{2}{*}{\multicolumn{2}{|c|}{8.195}} & \multirow{2}{*}{\multicolumn{2}{|c|}{$<0.05 a$}} \\
\hline No & 62 & 39 & 78.5 & 56.5 & & & & \\
\hline \multicolumn{9}{|l|}{ Indications } \\
\hline Cephalopelvic disproportion & 41 & 16 & 51.9 & 23.2 & 12.820 & \multirow{9}{*}{28.375} & $<0.01 a$ & \multirow{9}{*}{$<0.001 a$} \\
\hline Abnormal amniotic fluid & 9 & 8 & 11.4 & 11.6 & 0.001 & & 0.969 & \\
\hline Non-medical factors & 7 & 13 & 8.9 & 18.8 & 3.139 & & 0.076 & \\
\hline Fetal distress & 6 & 10 & 7.6 & 14.5 & 1.817 & & 0.178 & \\
\hline Breech presentation & 5 & 1 & 6.3 & 1.4 & 1.175 & & 0.278 & \\
\hline Nuchal cord & 4 & 6 & 5.1 & 8.7 & 0.303 & & 0.582 & \\
\hline Shoulder presentation & 4 & 0 & 5.1 & 0 & 1.923 & & 0.165 & \\
\hline Pregnancy complications & 2 & 11 & 2.5 & 15.9 & 8.267 & & $<0.01 a$ & \\
\hline Placental abnormalities & 1 & 4 & 1.3 & 5.6 & 1.137 & & 0.286 & \\
\hline
\end{tabular}

a $p<0.05$ 
and presence or absence of the first stage of labor in the previous cesarean section.

\section{Statistical analysis}

To find if there's any difference of the risk factors showed above, all the data were analyzed by chi-square test. A P-value $<0.05$ was considered statistically significant.

\section{RESULTS \\ Patient details}

As shown in Figure $1 \mathrm{~A}$, the uterus and cervical canal were empty and the gestational sac located in the anterior part of the isthmic portion of the uterus with a diminished myometrial layer between the bladder and the sac, which could not be shown by ultrasound examination (Fig. 1A) [18]. Intraoperative findings revealed that the bladder was densely adhered to the lower uterine segment and the gestational sac implanted through the cesarean scar. The bulging gestation sac appeared to be covered only by thin hypointense serosa and the left portion of the gestational sac had protruded through the thin hypointense serosa (Fig. 1B, C).

The mean maternal age was $32.7 \pm 5.1$ years (range: 21-43) in CSP group and $32.7 \pm 6.3$ years (range: $23-44$ ) in non-CSP group. No significant difference was noted between the two groups $(P>0.05)$. The gravidity ranged from 2 to 8 in CSP group and 2-6 in non-CSP group. All of the participants had delivered by at least 1 lower segment caesarean sections in both groups.

\section{Gravity history information analysis}

As shown in Table 1, 11.4\% (9 of 79) CSP patients had 2 or more previous cesarean sections, which was $13.0 \%$ (9 of 69) in non-CSP patients. No significant difference was noted between the two groups $(P>0.05)$. 87.3\% (69 of 79) CSP patients were more than 37 weeks gestational age before the last cesarean section, which was $88.4 \%$ (61 of 69) in the non-CSP group, the difference was not statistically different $(P>0.05)$.

In addition, we found that $22.8 \%$ (18 of 79) CSP patients had pregnancies for more than 5 times and $46.8 \%$ (37 of 79) had pregnancies for more than 4 times, which were $4.3 \%$ (3 of 69) and $10.1 \%$ (7 of 79 ) respectively in control group. 30.4\% (24 of 79) CSP patients had 3 pregnancies and 16.5\% (13 of 79) had 2 pregnancies, which were $26.1 \%$ ( 18 of 69 ) and $27.5 \%$ (19 of 79) respectively in control group. Significant difference was noted between the two groups $(P<0.001)$.

\section{Timing of the previous cesarean section}

As shown in Table 1, 41.8\% (33 of 79) and 58.2\% of CSP patients had undergone an emergency or elective surgery during the previous cesarean section respectively. Similarly, $37.7 \%$ (26 of 69 ) and $62.3 \%$ of non-CSP patients had undergone an emergency or elective surgery respectively. No significant difference was noted between the two groups $(P>0.05)$.

During the previous caesarean section, $21.5 \%$ (17 of 79) patients in CSP group entered the first stage of labor and showed cervical dilation and shortening before cesarean section, which was noted in $43.5 \%$ (30 of 69) in non-CSP group. Significant difference was noted between the two groups $(P<0.05)$.

\section{Indications for the previous cesarean section and possible risk factors for CSP}

The indications for the previous cesarean section were shown in Table 1. As shown in Table 1, cephalopelvic disproportion was the most common indication for cesarean sections in both groups, the rate of which were $51.9 \%$ (41 of 79) in CSP group and $23.2 \%$ (16 of 69) in non-CSP group. The incidence of cephalopelvic disproportion was higher in CSP
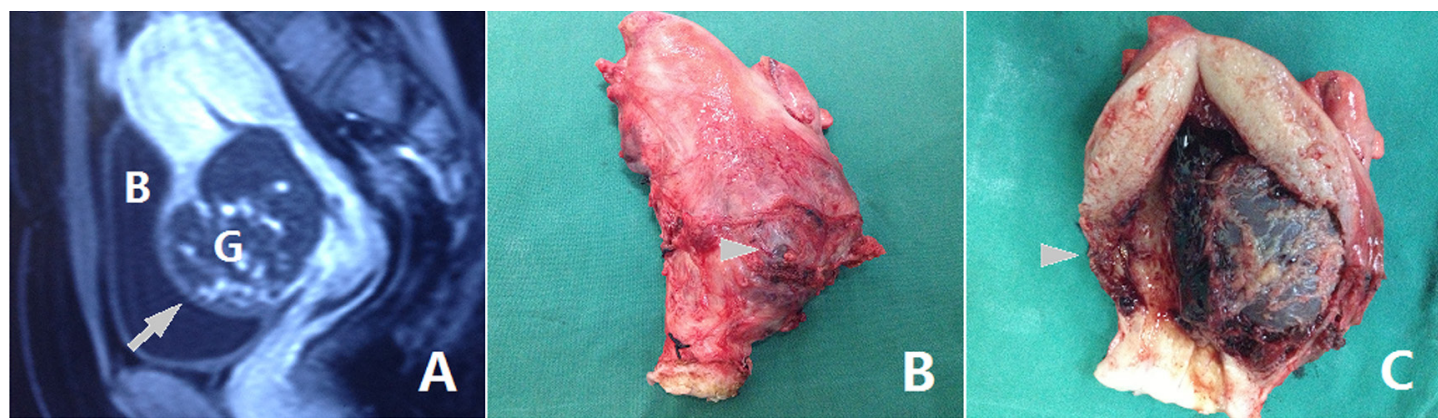

Figure 1. Cesarean scar pregnancy.

A. Cesarean scar pregnancy at 12 weeks in a woman with a history of multiple cesarean deliveries examined by MRI. Sagittal T1-weighted image of the pelvis shows an enlarged uterus and an outward bulging gestational sac $(G)$ within the anterior lower uterine segment. A marked thinned myometrium is seen between the gestational sac and bladder (B) with a suspicion of the placenta protruding through the serosa (arrow). The endometrial and cervical canals are empty. B, C. Cesarean scar pregnancy at 12 weeks in a woman who finally underwent a hysterectomy. Intraoperative findings revealed a very thin overlying myometrium. The bulging gestation sac appeared to be covered only by thin hypointense serosa and the left portion of the gestational sac had protruded through the thin hypointense serosa (arrow) 
group compared with non-CSP group (51.9\% vs $23.2 \%$ ). In addition to the leading indication for cesarean section, we also found that the incidence of breech and shoulder presentation was higher in CSP group compared with non-CSP group (11.4\% vs 1.4\%). However, the incidence of indications including non-medical factors, fetal distress, pregnancy complications, and placental abnormalities were lower in CSP group compared with non-CSP group (Tab. 1).

Using Chi-square test, we analyzed the possible risk factors for CSP.The data showed that the number of pregnancies was statistically different between CSP group and non-CSP group $(P<0.001)$. Fewer patients in CSP-group had entered the first stage of labor during the previous caesarean delivery compared with non-CSP group $(P<0.05)$. The indications of cesarean sections were significantly different between the two groups $(P<0.001)$. The maternal age, multiple cesarean sections, gestational age, emergency or elective caesarean section showed no significant difference between the two groups $(P>0.05)$. More cephalopelvic disproportion was noted in CSP patients $(P<0.01)$. Although the incidence of breech and shoulder presentation was higher in CSP group compared with non-CSP group (11.4\% vs 1.4\%), no significant difference was noted $(P>0.05)$. In addition, no significant difference of the incidence of indications including abnormal amniotic fluid, non-medical factors, fetal distress, nuchal cord, and placental abnormalities were found between CSP group and non-CSP group (Tab. 1, $P>0.05$ ).

\section{DISCUSSION}

The clinical presentation of CSP was nonspecific at presentation and the common symptom was vaginal bleeding with or without abdominal pain [19]. Some patients had heavy bleeding during or after dilatation and curettage. The most common method in detecting CSP is transvaginal sonographic examination and magnetic resonance imaging (MRI). MRI is useful as a troubleshooting tool and can better assess the possibility of myometrial invasion and bladder involvement, which can provide additional information for directing therapy $[8,20,21]$.

In the current study, no significant difference of ages was noted between CSP patients and non-CSP group, indicating that maternal age might not be involved in the occurrence of CSP. Similarly, the gestational age during the previous caesarean section showed no significant difference between the two groups.

Previous studies reported that the presence of cesarean scar (CS) affected future implantation, and there were significant differences in placental location between the CS and non-CS groups. The gestation sac implantation was most posterior and lower in the CSP group and fundal in the non-CS group $[22,23]$. Gestation sac implantation was significantly lower in the CS group [23]. The presence of a CS scar may lead to a change in myometrial contractility and damage the integrity of the myometrial-endometrial junctionalzone.

Jurkovic et al. [9] reported that $72 \%$ of their CSP patients had undergone multiple $(\geq 2)$ caesareans and they considered multiple previous caesareans a risk factor of CSP. The limitation of their studies was sample size, which was only 18 CSP patients in their study. However, Chuang et al. [24] and Maymon et al. [25] found that the number of previous caesareans might not be a risk factor. In consistence with Chuang et al and Maymon et al studies [24, 25], we found that only $11.4 \%$ of CSP patients had undergone caesareans for more 2 times which was $13.0 \%$ in non-CSP group. Multiple caesareans might not be a risk factor for the occurrence of CSP, although multiple caesareans may increase scar surface area.

On the contrary, we found that CSP group had more previous pregnancies compared with non-CSP group. The data indicated that multiple pregnancies may be a risk factor for the occurrence of CSP. Multiple pregnancies may result in endometrial or muscular injuries, especially when dilatation and curettage is used to terminate the pregnancy.

We found that only $21.5 \%$ of CSP patients entered the first stage of labor before the previous caesarean section, which was significantly lower compared with that of non-CSP group. These data indicated that absence of the first stage of labor might be a risk factor for the occurrence of CSP. During the first stage of labor, with the regular uterine contractions, the effacement and dilatation of cervix happened. When the operations were performed before entering the first stage of labor and in a non-developed lower uterine segment, the cesarean incision site might be imprecise. Finally, Maymom et al found that the healing processes following the operations might facilitate implantation of the blastocyst within the scar $[7,8]$.

In the current study, cephalopelvic disproportion was the most common indication of caesarean section. More than $50 \%$ of CSP patients had underwent caesarean section due to cephalopelvic disproportion during the previous caesarean section, which was significantly higher compared with non-CSP group. In addition, more breech presentation and shoulder presentation were noted in CSP group compared with non-CSP group, indicating that breech presentation and shoulder presentation might be risk factors of the occurrence of CSP. The mechanism underlying the correlation between indications for caesarean section and CSP is that cephalopelvic disproportion, breech presentation and shoulder presentation can cause a non-developed lower uterine segment.

In the current study, the cesarean sections included emergency surgeries and elective surgeries. Many expectant women chose elective surgeries for complications or non-medical reasons while others indeed need an emergency 
cesarean section due to medical reasons, including serious complications. The distribution of the two surgeries showed no significant difference between CSP group and non-CSP group. The data suggest that the indication of the surgery but not the types of the surgeries is the risk factor for the occurrence of CSP.

Taken together, multiple pregnancies, absence of first stage of labor, the indications of the previous caesarean section, might be risk factors for the occurrence of CSP. Conversely, maternal age, multiple cesarean sections, gestational age, emergency or elective caesarean section showed no significant difference between CSP group and non-CSP group.

The difference in surgical technique might also be invoIved in the occurrence of CSP. However, a larger series study would be needed to further elucidate these issues. This is also the main limitation of this study due to its retrospective design.

The rapid increasing number of patients with scar pregnancy in China reflects that caesarean sections are being widely performed due to medical or non-medical reasons. The decrease in use of the unnecessary caesarean sections will be an effective way to reduce the incidence of CSP.

This study was conducted in accordance with the declaration of Helsinki and conducted with approval from the Ethics Committee of Shandong University with written informed consent was obtained from all participants.

\section{Acknowledgements}

The authors would like to acknowledge the contributions of Jifeng Bian, who helped a lot in the edit of the manuscript. The research was supported by grants from the National Natural Science Foundation of China (No. 81270661, No. 81300468) and a grant from Shandong Province excellent youth scientist foundation (No. BS2013YY008).

\section{Conflict of interest}

The authors have no competing interests to declare.

\section{REFERENCES}

1. Larsen JV, Solomon MH. Pregnancy in a uterine scar sacculus--an unusual cause of postabortal haemorrhage. A case report. S Afr Med J. 1978; 53(4): 142-143, indexed in Pubmed: 653492.

2. Fylstra DL. Ectopic pregnancy within a cesarean scar: a review. Obstet Gynecol Surv. 2002; 57(8): 537-543, doi: 10.1097/01. OGX.0000025517.33346.1E, indexed in Pubmed: 12187153.

3. Riaz RM, Williams TR, Craig BM, et al. Cesarean scar ectopic pregnancy: imaging features, current treatment options, and clinical outcomes. Abdom Imaging. 2015; 40(7): 2589-2599, doi: 10.1007/s00261-015-0472-2, indexed in Pubmed: 26070747

4. Ugurlucan FG, Bastu E, Dogan M, et al. Management of cesarean heterotopic pregnancy with transvaginal ultrasound-guided potassium chloride injection and gestational sac aspiration, and review of the literature. J Minim Invasive Gynecol. 2012; 19(5):671-673, doi: 10.1016/j. jmig.2012.05.006, indexed in Pubmed: 22935313.

5. Uysal F, Uysal A, Adam G. Cesarean scar pregnancy: diagnosis, management, and follow-up. J Ultrasound Med. 2013; 32(7): 1295-1300, doi: 10.7863/ultra.32.7.1295, indexed in Pubmed: 23804353.
6. Birge Ö, Karaca C, Arslan D, et al. Medical management of cesarean scar pregnancy at advanced age: case report and literature review. Clin Exp Obstet Gynecol. 2016; 43(1): 140-142, indexed in Pubmed: 27048038.

7. Naji O, Daemen A, Smith A, et al. Changes in Cesarean section scar dimensions during pregnancy: a prospective longitudinal study. Ultrasound Obstet Gynecol. 2013; 41(5): 556-562, doi: 10.1002/uog.12334, indexed in Pubmed: 23108803.

8. Maymon R, Halperin R, Mendlovic S, et al. Ectopic pregnancies in a Caesarean scar: review of the medical approach to an iatrogenic complication. Hum Reprod Update. 2004; 10(6): 515-523, doi: 10.1093/humupd/dmh042, indexed in Pubmed: 15375087.

9. Jurkovic D, Hillaby K, Woelfer B, et al. Cesarean scar pregnancy. Ultrasound Obstet Gynecol. 2003; 21(3): 310, doi: 10.1002/uog.55, indexed in Pubmed: 12666232

10. Wang $\mathrm{Q}$, Peng $\mathrm{HL}, \mathrm{He} \mathrm{L}$, et al. Reproductive outcomes after previous cesarean scar pregnancy: Follow up of 189 women. Taiwan J Obstet Gynecol. 2015; 54(5): 551-553, doi: 10.1016/j.tjog.2015.08.006, indexed in Pubmed: 26522109.

11. Seow KM, Huang LW, Lin YH, et al. Cesarean scar pregnancy: issues in management. Ultrasound Obstet Gynecol. 2004; 23(3): 247-253, doi: 10.1002/uog.974, indexed in Pubmed: 15027012.

12. Lincenberg KR, Behrman ER, Bembry JS, et al. Uterine Rupture with Cesarean Scar Heterotopic Pregnancy with Survival of the Intrauterine Twin. Case Rep Obstet Gynecol. 2016; 2016: 6832094, doi: 10.1155/2016/6832094, indexed in Pubmed: 28116191.

13. Mahajan D, Kang M, Sandhu MS, et al. Rare complications of cesarean scar Indian J Radiol Imaging. 2013; 23(3): 258-261, doi: 10.4103/09713026.120265, indexed in Pubmed: 24347858.

14. Api M, Boza A, Gorgen H, et al. Should Cesarean Scar Defect Be Treated Laparoscopically? A Case Report and Review of the Literature. J Minim Invasive Gynecol. 2015; 22(7): 1145-1152, doi: 10.1016/j.jmig.2015.06.013, indexed in Pubmed: 26122897.

15. Qian ZD, Guo QY, Huang LL. Identifying risk factors for recurrent cesarean scar pregnancy: a case-control study. Fertil Steril. 2014; 102(1): 129-134.e1, doi: 10.1016/j.fertnstert.2014.04.003, indexed in Pubmed: 24825421.

16. Vial $Y$, Petignat $P$, Hohlfeld P. Pregnancy in a cesarean scar. Ultrasound Obstet Gynecol. 2000; 16(6): 592-593, doi: 10.1046/j.1469-0705.2000.00300-2.x, indexed in Pubmed: 11169360.

17. Marchiolé $P$, Gorlero F, de Caro G, et al. Intramural pregnancy embedded in a previous Cesarean section scar treated conservatively. Ultrasound Obstet Gynecol. 2004; 23(3): 307-309, doi: 10.1002/uog.981, indexed in Pubmed: 15027026

18. Wu R, Klein MA, Mahboob S, et al. Magnetic resonance imaging as an adjunct to ultrasound in evaluating cesarean scar ectopic pregnancy. J Clin Imaging Sci. 2013; 3: 16, doi: 10.4103/2156-7514.109758, indexed in Pubmed: 23814688.

19. Rotas MA, Haberman S, Levgur M. Cesarean scar ectopic pregnancies: etiology, diagnosis, and management. Obstet Gynecol. 2006; 107(6): 1373-1381, doi: 10.1097/01.AOG.0000218690.24494.ce, indexed in Pubmed: 16738166.

20. Ash A, Smith A, Maxwell D. Caesarean scar pregnancy. BJOG. 2007; 114(3): 253-263, doi: 10.1111/j.1471-0528.2006.01237.x, indexed in Pubmed: 17313383

21. Rosen T. Placenta accreta and cesarean scar pregnancy: overlooked costs of the rising cesarean section rate. Clin Perinatol. 2008; 35(3): 519-529,, doi: 10.1016/j.clp.2008.07.003, indexed in Pubmed: 18952019.

22. Naji O, Wynants L, Smith A, et al. Does the presence of a Caesarean section scar affect implantation site and early pregnancy outcome in women attending an early pregnancy assessment unit? Hum Reprod. 2013; 28(6): 1489-1496, doi: 10.1093/humrep/det110, indexed in Pubmed: 23585560.

23. Naji O, Daemen A, Smith A, et al. Does the presence of a cesarean section scar influence the site of placental implantation and subsequent migration in future pregnancies: a prospective case-control study. Ultrasound Obstet Gynecol. 2012; 40(5):557-561, doi: 10.1002/uog.11133, indexed in Pubmed: 22323094.

24. Chuang J, Seow KM, Cheng WC, et al. Conservative treatment of ectopic pregnancy in a caesarean section scar. BJOG. 2003; 110(9): 869-870, indexed in Pubmed: 14511972.

25. Maymon R, Halperin R, Mendlovic S, et al. Ectopic pregnancies in Caesarean section scars: the 8 year experience of one medical centre. Hum Reprod. 2004; 19(2): 278-284, indexed in Pubmed: 14747167. 